\title{
Immunonutrition in radical cystectomy: a novel approach to reduce postoperative complications?
}

\author{
Atiqullah Aziz, Oliver W. Hakenberg \\ Department of Urology, University Medical Center Rostock, Rostock, Germany \\ Correspondence to: Atiqullah Aziz, MD, FEBU, MBA. Department of Urology, University Medical Center Rostock, Ernst-Heydemann-Str. 6, 18057 \\ Rostock, Germany. Email: atiqullah.aziz@med.uni-rostock.de. \\ Provenance: This is an invited Editorial commissioned by Section Editor Xiao Li (Department of Urology, Jiangsu Cancer Hospital \& Jiangsu \\ Institute of Cancer Research \& Nanjing Medical University Affiliated Cancer Hospital, Nanjing, China). \\ Comment on: Hamilton-Reeves JM, Stanley A, Bechtel MD, et al. Perioperative Immunonutrition Modulates Inflammatory Response after Radical \\ Cystectomy: Results of a Pilot Randomized Controlled Clinical Trial. J Urol 2018;200:292-301.
}

Submitted Oct 05, 2018. Accepted for publication Oct 12, 2018.

doi: $10.21037 /$ tau.2018.10.06

View this article at: http://dx.doi.org/10.21037/tau.2018.10.06

Despite improvements in perioperative management, radical cystectomy $(\mathrm{RC})$ remains still associated with mortality rates of up to $9 \%$ within 90 days after surgery across all age groups (1). With this in mind, attempts to minimize postoperative complications are essential.

Compared to other disciplines such as gastrointestinal surgery (2), the influence of the patient's nutritional status on the outcome of RC has not been extensively studied. However, since nutritional status is assumed to influence postoperative morbidity, the authors of the randomized controlled clinical trial published in this issue are to be commended for their pioneering work (3). HamiltonReeves et al. comparatively analyzed a total of 29 men that were randomly assigned to receive either a Specialized IMmunonutrition (SIM; L-arginine, omega-3 fatty acids, dietary nucleotides; $\mathrm{n}=14$ ) or a standard oral nutrition supplement without immunomodulators (ONS; $\mathrm{n}=15)$ for five days pre- and postoperatively, respectively, in order to evaluate the impact on acute inflammatory response (IR) and arginine status after RC. Using T-helper (Th)1-Th2 balance as a surrogate for the IR, the authors found that the intake of this SIM had a significant impact on the Th1-Th2 balance which was shifted towards a Th1 response with the SIM compared to ONS. Thus, with immunonutrition patients may benefit since a shift toward a predominant Th1 response is perhaps more likely to be associated with less postoperative complications (4). The findings of the current SIM study on RC are in line with findings from large randomized control trials in gastrointestinal surgery $(5-7)$ that the intake of SIM counteracts Th1-Th2 imbalance after major surgery.

Also, IL-6 levels were lower in the SIM group compared to ONS showing a reduced IR on the second postoperative day. Other data from studies on hepatobiliary surgery also support this finding of a reduced IL-6 level with immunonutrition, which might be protective against increased muscle catabolism and complications $(8,9)$. The intake of SIM was also associated with higher serum ornithine levels 30 days after surgery compared to ONS. Ornithine potentially has a beneficial effect on would healing with increased collagen formation due higher proline and polyamine availability (10). SIM also seemed to lead to less pronounced muscle loss two weeks after RC. Interestingly, the serum level of C-reactive protein was not significantly different between SIM and ONS.

Half of the patients in the SIM group received neoadjuvant chemotherapy which in itself may lead to an impaired nutritional status. Immunonutrition might be a beneficial early intervention to avoid malnutrition and consecutive muscle loss with added chemotherapy (11).

Enhanced Recovery After Surgery (ERAS) protocols are currently widely used in RC patients, with a better and more standardized perioperative care, earlier recovery and less complications (12). ERAS was not included in the current study nor was a standardized assessment of complication. Thus, comparing the findings of this study to current practice patterns is not possible.

Overall, although the findings are of high interest, 
the small sample size, the short follow-up and the nonstandardized design in some respects make this study, although an RCT, a pilot study. Further larger and more elaborate studies are needed as many of the findings in this small trial may be incidental and the interpretations are still largely hypothetical. The underlying hypothesis, that immunonutrition may well improve results of $\mathrm{RC}$ is extremely interesting.

\section{Acknowledgements}

None.

\section{Footnote}

Conflicts of Interest: The authors have no conflicts of interest to declare.

\section{References}

1. Aziz A, May M, Burger M, et al. Prediction of 90-day mortality after radical cystectomy for bladder cancer in a prospective European multicenter cohort. Eur Urol 2014;66:156-63.

2. Burden S, Todd C, Hill J, et al. Pre-operative nutrition support in patients undergoing gastrointestinal surgery. Cochrane Database Syst Rev 2012;11:CD008879.

3. Hamilton-Reeves JM, Stanley A, Bechtel MD, et al. Perioperative Immunonutrition Modulates Inflammatory Response after Radical Cystectomy: Results of a Pilot Randomized Controlled Clinical Trial. J Urol 2018;200:292-301.

4. Marik PE, Flemmer M. The immune response to surgery and trauma: Implications for treatment. J Trauma Acute Care Surg 2012;73:801-8.

Cite this article as: Aziz A, Hakenberg OW. Immunonutrition in radical cystectomy: a novel approach to reduce postoperative complications? Transl Androl Urol 2018;7(Suppl 6):S718-S719. doi: $10.21037 /$ tau.2018.10.06
5. Matsuda A, Furukawa K, Takasaki H, et al. Preoperative oral immune-enhancing nutritional supplementation corrects TH1/TH2 imbalance in patients undergoing elective surgery for colorectal cancer. Dis Colon Rectum 2006;49:507-16.

6. Suzuki D, Furukawa K, Kimura F, et al. Effects of perioperative immunonutrition on cell-mediated immunity, T helper type 1 (Th1)/Th2 differentiation, and Th17 response after pancreaticoduodenectomy. Surgery 2010;148:573-81.

7. Aida T, Furukawa K, Suzuki D, et al. Preoperative immunonutrition decreases postoperative complications by modulating prostaglandin E2 production and T-cell differentiation in patients undergoing pancreatoduodenectomy. Surgery 2014;155:124-33.

8. Hübner M, Cerantola Y, Grass F, et al. Preoperative immunonutrition in patients at nutritional risk: results of a double-blinded randomized clinical trial. Eur J Clin Nutr 2012;66:850-5.

9. Uno H, Furukawa K, Suzuki D, et al. Immunonutrition suppresses acute inflammatory responses through modulation of resolvin $\mathrm{E} 1$ in patients undergoing major hepatobiliary resection. Surgery 2016;160:228-36.

10. Tobert CM, Mott SL, Nepple KG. Malnutrition Diagnosis during Adult Inpatient Hospitalizations: Analysis of a Multi-Institutional Collaborative Database of Academic Medical Centers. J Acad Nutr Diet 2018;118:125-31.

11. Tobert CM, Hamilton-Reeves JM, Norian LA, et al. Emerging Impact of Malnutrition on Surgical Patients: Literature Review and Potential Implications for Cystectomy in Bladder Cancer. J Urol 2017;198:511-9.

12. Pang KH, Groves R, Venugopal S, et al. Prospective Implementation of Enhanced Recovery After Surgery Protocols to Radical Cystectomy. Eur Urol 2017. [Epub ahead of print]. 